\title{
Preparation and characterization of floating porous graphite carbon monoliths produced from biomass and its application
}

\author{
Hanyu Chen ${ }^{1, *}$, Liangbo Zhang ${ }^{l}$, Baozhong Zhang ${ }^{l}$, Peiyu Duan ${ }^{1}$ \\ ${ }^{1}$ School of Environmental Engineering, Henan University of Technology, Zhengzhou, 450052, China
}

\begin{abstract}
Herein, a new floating porous graphite carbon monolith (FCM) was prepared using the graphite powder and peanut shells as the carbon skeleton and using FAC and sepiolite as the swelling agent. The multi-hierarchical porous FCMs were obtained by two-step acid activation method. The effects of the addition of biomass and phosphoric acid on the physical properties and pore structure of the prepared material were investigated. The FCM was combined with codoped titania to develop a new floating photocatalysts. The results showed an increase in the amount of biomass materials during FCM preparation increased the ratio of mesoporous structure in the products. In addition, a high micropore volume ratio and the strongest adsorption effect were achieved by optimizing the phosphoric acid impregnation ratio. After process optimization, the densities of the prepared FCMs ranged between 0.75 and $0.89 \mathrm{~g} / \mathrm{cm} 3$, and the specific surface area and diameter range of the large pores of the material were $104.5 \mathrm{~m} 2 / \mathrm{g}$ and 3-50 $\mu \mathrm{m}$, respectively. Moreover, the new floating catalysts displayed the excellent photocatalytic properties and recyclability performance.
\end{abstract}

\section{Introduction}

Because of its good porous and good surface properties, shaped macro/mesoporous activated carbon is already used for environmental restoration, gas storage and capture, energy storage and conversion and catalyst carriers [1-3]. With the increasing number of publications on shaped carbon materials, the application of active carbon monoliths(ACMs) have been a hot topic in recent years [4-5]. With regard to their material characteristics, ACMs exhibit a large proportion of extensive pore structures, a high chemical stability, a large specific surface area and good adsorption capacity. ACMs have outstanding performance as adsorbents and catalyst carriers. In addition, ACMs possess significant advantages in terms of the filling, transportation and reuse of materials compared with amorphous carbon materials [6-9].

ACMs which is prepared by using environmentally friendly processes and low-cost biomass, has been favored by researchers both in China and abroad. Template synthesis is a commonly adopted method for the preparation of hierarchically macro-mesoporous carbon materials. Biomass materials need to be mixed with other silicon-based templates to enhance their strength and formability in preparation processing. However, most of the researches idea were limited on the preparation of lab-scale shaped carbon in the design processing. Only a few of studies have been conducted on the preparation of large-scale shaped carbon monoliths directly from biomass. At present, large size shaped macro/mesoporous carbon monoliths are primarily prepared by extrusion molding, and the raw materials used for the preparation of these ACMs include activated carbon powder, micellar carbon fibers and carbonized resin [10-12]. In addition, the binders used for the preparation of ACMs include various organic and inorganic binders, including coal tar, pitch, phosphoric acid and polyvinyl alcohol [13-15]. Extruded ACMs form granules or cake-shaped materials [16].

Surface water pollution is a popular question with the development of society industrialization. Thus, the green renewable surface water purification technology which can efficiently treatment surface water is greatly needed. Floating macro-mesoporous carbon materials are promising candidates for its adsorption and catalytic degradation technology applications in surface water purification. Several types of macro-mesoporous carbon materials with good floatability have already been studied and used to improve the photocatalytic activity of the catalyst. These materials include expanded graphite, spongy graphite materials and magnetically separated porous carbon materials [17-19]. However, these materials have multiple disadvantages. The synthesis of these materials is costly and requires relatively complicated steps. Furthermore, these materials have low strength and cannot be recycled effectively.

Peanut shells could form honeycomb pores and lamellar structures by activated with phosphoric acid. The preparation of activated carbon blocks from activated peanut shell has been reported [20]. The cenosphere separated from fly-ash used as a type of economic source has been applied on the photocatalytic

\footnotetext{
* Corresponding author: Tel./Fax: +86 0371-67756718; chypds@126.com (H Y Chen)
} 
technology in the field of sewage treatment due to its floating property [21]. Our group has also studied the degradation of pollutants using magnetic floating beads supported catalysts [22]. Graphite powder as a solid waste resource could improve the product strength due to its resistance against oxidation at high temperatures. In this study, a one pot process for the preparation of floating porous carbon monolith using biomass materials has been developed. The process utilizes solid wastes, such as peanut shells, graphite electrode powder and cenosphere, as raw materials to prepare a new type of floating carbon material.

This study aims to provide a cost-effective and convenient strategy for the production of floating ACMs that can be used in water purification and heterogeneous catalysis fields. In addition, the production of these ACMs is a green process that minimizes the quantity of required chemicals and converts waste materials into useful products. At present, the preparation of such materials has not yet been reported in the literature.

\section{Materials and methods}

\subsection{Study materials}

Peanut shells were purchased from the local market. Graphite powder (particles with a size range of $65-75 \mu \mathrm{m}$ ) was obtained from a discarded graphite electrode. Prior to use, the graphite powder was pulverized and dried. Fly ash cenosphere (FAC) particles with a size range of 80-125 $\mu \mathrm{m}$ were obtained from Pingdingshan Yaomeng Thermal Insulation Material Co., Ltd. (Henan, China). The particles floating on the surface of the solution were collected and dried in an oven at $105{ }^{\circ} \mathrm{C}$ for $24 \mathrm{~h}$. The dried FAC particles were rinsed with $0.1 \mathrm{M}$ nitric acid and washed in boiling deionized water until a solution $\mathrm{pH}$ value of 7 was achieved. Polyethylene glycol (PEG400 , GR) was obtained from Shanghai Guoyao Regents Co. (Shanghai, China).

\subsection{Synthesis of FCMs}

A certain amount of epoxy resin, pulverized peanut shells and graphite powder were mixed together. Phosphoric acid solutions with different weight ratios were then added to activate the carbon material at $80^{\circ} \mathrm{C}$. When the color of the mixture turned from brown to black and the mixture became highly viscous, an appropriate amount of polyvinyl alcohol solution was added to the mixture and dissolved by heating. FAC and a certain amount of sepiolite powder were added to the mixtures before they were heated and stirred at $60{ }^{\circ} \mathrm{C}$ in a water bath held at a constant temperature. Once a viscous and moldable material was obtained, the mixture was loaded into a cylinder-shaped mold and cold-pressed. After molding, the material was dried at $110^{\circ} \mathrm{C}$ for three hours in an oven. After the samples were dried, they were placed in a vacuum tube furnace used for carbonization. The FCMs were produced by heating the spheroid-shaped material at $550{ }^{\circ} \mathrm{C}$ for 150 min under a continuous flow of $\mathrm{N}_{2}(500 \mathrm{~mL} / \mathrm{min})$. After the materials cooled to room temperature, they were dipped into the phosphoric acid solutions for secondary acid activation 6-8 h. After that, the FCMs washed using water and sonication until their $\mathrm{pH}$ value became neutral. Subsequently, the properties of the materials were tested.

\subsection{Characterization of the FCMs}

The specific surface area, pore volume and pore size distribution of the materials were measured with $\mathrm{N}_{2}$ adsorption-desorption isotherms created using a V-Sorb analyser.(V-2800TP-Ginger spectrum, Beijing,China). The morphology and particle size of the resulting materials were examined using a Phenom-Prox scanning electron microscope (SEM). X-ray diffraction (XRD) was performed using a Bruker D8 Advance (German) powder diffractometer $(\mathrm{Cu} \mathrm{K \alpha}$ radiation, $\lambda=1.5406 \AA)$. Fourier transform infrared (FTIR) spectra were recorded on a Nicolet 5700 FTIR spectrometer using conventional $\mathrm{KBr}$ pellets. Other properties such as bulk density, ash content, compressive strength and the methylene blue uptake of samples were tested using the methods described in [23].

\subsection{The preparation of FCMs carried photo catalyst}

The floating photocatalyst was prepared by N-S-TiO immobilized on FCMs as below. The FCMs was crushed and the fragments washed with distilled water $(\mathrm{pH} 7.2)$ and then dried at about $100{ }^{\circ} \mathrm{C}$ for $1 \mathrm{~h}$. N-S-TiO composite membranes were prepared via sol-gel method. In $20 \mathrm{~mL}$ dry ethanol, $10 \mathrm{~mL}$ tetrabutyl titanate was dissolved to obtain solution A. Thiourea solution $(3 \mathrm{~mL}$ acetic acid and $0.782 \mathrm{~g}$ salt) was then dissolved with 30 $\mathrm{mL}$ dry ethanol, to obtain the homogeneous solution B. The $\mathrm{pH}$ of the solution was adjusted to $2-3$ by nitric acid $\left(1 \mathrm{~mol} \mathrm{~L}^{-1}\right)$. Thereafter, solution A was added dropwise into solution B under strong stirring for $2 \mathrm{~h}$ to obtain the sol. The obtained sol was left at room temperature for 6$8 \mathrm{~h}$, then the FCMs pieces were dipped in the sol for two times then dry in the air to get the gel. Next, the gel was calcined at $450{ }^{\circ} \mathrm{C}$ in static air for $2 \mathrm{~h}$ to obtain white photocatalyst composite membranes. Columnar activated carbon and graphite pieces were also used as substrates to prepare immobilized photocatalyst by the same processing for comparison with FCMs material.

\section{Results and Discussion}

In this study, graphite powder and peanut shells were used to form the carbon skeleton of shaped porous carbon. Desirable outer dimensions and mechanical properties were obtained by controlling the pore size of the shaped carbon via phosphoric acid activation (Fig. 1a). Photographs of the as-synthesized and corresponding carbon monoliths are shown in Fig. 1a and $1 \mathrm{~b}$, respectively. Highly stable carbon samples that retained a monolithic shape were obtained after carbonization. 
The addition of FAC and sepiolite to shaped carbon blocks was performed to improve the floatability of porous carbon. In addition, to further control the bulk density of the material, a small amount of epoxy resin was added to the raw material as a swelling agent. As a result, a shaped macro/mesoporous carbon composite material with a low density (Fig. 1b) was obtained.

Although the volume of the carbide increased during the carbonization process from reacting with polyvinyl alcohol and resin, the carbon blocks remained crack-free after carbonization. The samples sustaining a pressure of 2.3-4.02 MPa in the tests indicated that they could

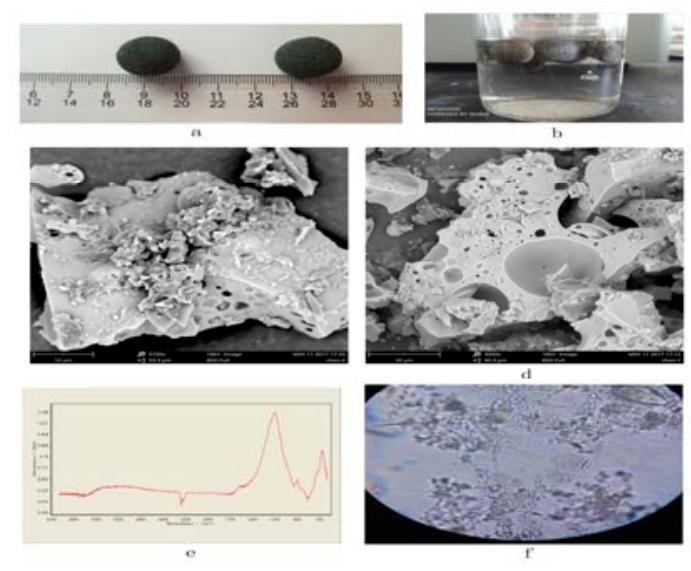

Fig. 1. Photographs of (a) monoliths sizes and (b) floating carbon samples in water, SEM images of (c) the pore size distributions of monoliths without sepiolite and (d) a swelled sample. (e) FTIR spectrum of the GCM sample. (f)

micrographs of monoliths containing microorganisms.

withstand high-pressure conditions. Moreover, they retained their intact structure after $45 \mathrm{~d}$ of stirring in the designed water treatment reactor and, after being dried and activated, could be reused for six times used as load carrier, the photocatalist still keeping high catalytic activity. These results indicated this material retained its intact structure after multiple times of stirring and rinses.

The internal structural information of the material is shown in Fig. 1c and d. These images show the porous structures of the carbon block with or without addition of the swelling agent. When swelling agent was added, large pores formed inside the material. When no swelling agent was added, the inside of the synthesized carbon block has a honeycomb fiber structure. The honeycomb fiber structure typically manifested as a porous material formed using lignocellulosic materials.

Plant material was used as a precursor for carbonbased materials, and it contained a large number of hydroxyl groups. As shown in Fig. 1a and e and according to the results obtained from the infrared database, the absorption peak at $2375 \mathrm{~cm}^{-1}$ corresponded to the carboxyl group and the absorption peak at 1100 $\mathrm{cm}^{-1}$ corresponded to the hydroxyl group. These hydroxyl groups significantly improved the water wettability of the prepared material. Therefore, the prepared porous carbon material was easily bound to, absorbed to and enriched by water-soluble organic pollutants. Its wettability made it a suitable material for use in water purification and pollutant adsorption.
Moreover, once inoculated and cultured, microorganisms were loaded onto the surface of the composite material, which functioned as a microorganism carrier (Fig. 1f).

Figure 2 showed the $\mathrm{N}_{2}$ adsorption-desorption isotherm obtained from the FCMs. The isotherm showed that the material was a combination of types I and IV. At relative low pressure $(<0.2)$, the isotherm exhibits a low adsorption, indicating the samples contain amorphous carbon microspores (type I). At the high relative pressure range of $0.4-1$, the curve exhibits obvious hysteresis loop, indicating the pore-size distributions in the mesoporous and macroporous region. And the dates were determined that the solid BET surface areas are in the between of 32.7 and $104.2 \mathrm{~m}^{2} \mathrm{~g}^{-1}$, and the $\mathrm{Vt}$ value in the between of 0.055 and $0.13 \mathrm{~cm}^{3} \mathrm{~g}^{-1}$. The specific surface area of the floating carbon composite material was smaller than that of the porous carbon material prepared using peanut shells in [24]. However, the macroporous and mesoporous structures of the floating carbon composite material were more suitable for biofilms and nanocatalysts.

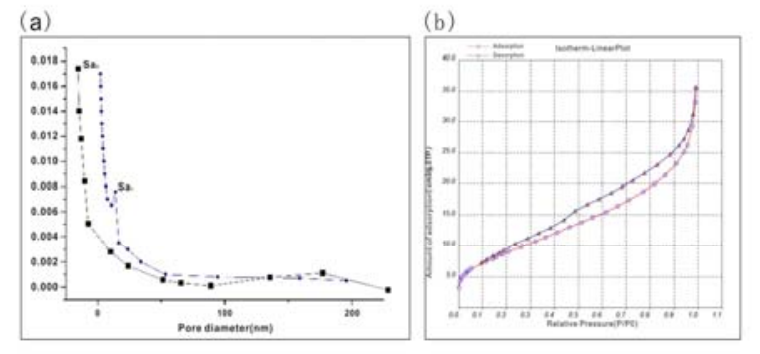

Fig. 2. (a) Adsorption isotherm-derived pore size distributions of floating carbon Samples 3 and 4. (b) $\mathrm{N}_{2}$ adsorptiondesorption isotherms.

The developed material was also used as substrate carrier with $\mathrm{N} \backslash \mathrm{S}$-codoped titania to prepare a floating photocatalytic material. SEM was used to directly observe the mesostructure of carbon materials and the distribution state of $\mathrm{N}-\mathrm{S}-\mathrm{TiO}_{2}$ nanoparticles on the surfaces of the FCMs and the columnar activated carbon. From the SEM image (Fig. $3 \mathrm{a}-\mathrm{c}$ ), $\mathrm{TiO}_{2}$ films were found on the outer layer of the graphite materials(3a), most amorphous particles were observed on the surface of the columnar activated carbon $(3 \mathrm{c})$. However, when the same photocatalysts load onto the surface of the FCMs material (3b), the nano-N-S- $\mathrm{TiO}_{2}$ catalyst became evenly distributed on the carrier material's surface. Inside the pores, the catalyst's particles were in a lamellar structure. This is because the peanut shell and resin were changed to the active carbon when it was calcined at $450^{\circ} \mathrm{C}$ to form the anisotropic porous carbon layer, after adding graphite and FAC microspheres, the graphite particles still provied the neat interior space. Compared to the columnar activated carbon (3c), a more uniform catalyst coating was formed on the developed carrier. The XRD patterns of the carrier and catalyst crystal structures are presented in Fig 3d. It can be seen that several broad peaks at $2 \theta=25.3,37.5,48.1$, and 54.00 , respectively, corresponding to (101), (004), (200), and (105/211) plane diffraction of anatase $\mathrm{TiO}_{2}$ are observed. With the addition of the thiourea and graphite, the intensities and 
widths of anatase diffraction peaks increase and become narrow, respectively, implying the enhancement of crystallization and growth of crystallites.

\section{Conclusions}

In this study, floating shaped porous FCMs with various pore sizes (macroporous, mesoporous and microporous) and good adsorption performance were prepared using the graphite powder and peanut shells as the carbon skeleton and using FAC and sepiolite as the swelling agent. The resulting material exhibited adequate strength
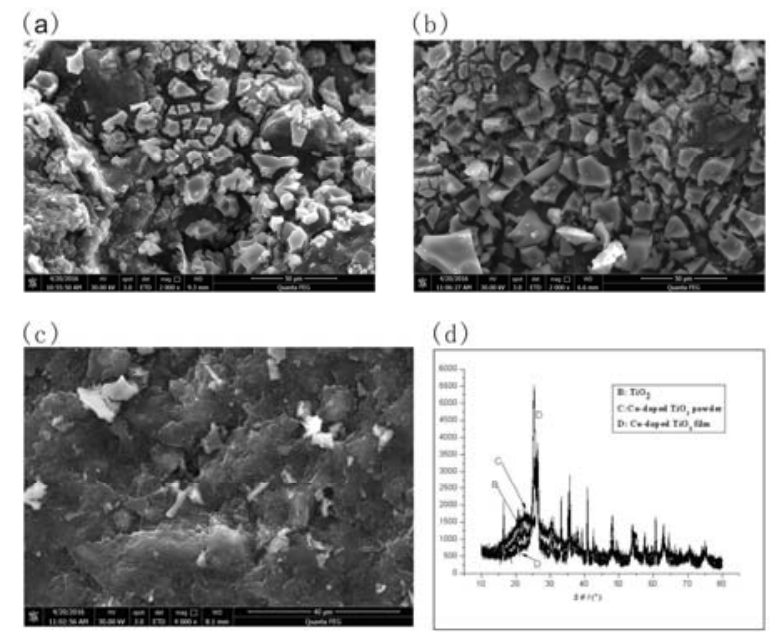

Fig.3. SEM images of (a) the sample used to determine the particle distribution on the FCM surface, (b) the lamellar structure of the catalyst and (c) the catalyst distribution on the surface of the columnar-activated carbon. (d) XRD spectra of the $\mathrm{N}-\mathrm{S}-\mathrm{TiO}_{2}$ nanoparticles.

and regular dimensions. The pore size of the material made it suitable for use as a carrier for catalysts and microorganisms. In addition, the physical properties of the new material were desirable for its use in environment with either a strong flushing force or a high pressure. FTIR characterization of the material and the wettability of the material in water indicated good water wettability. A floating carbon material with a density of $0.76 \mathrm{~g} / \mathrm{cm}^{3}$, a pressure resistance of $3.9 \mathrm{MPa}$ and a specific surface area of $32.7-104.2 \mathrm{~m}^{2} / \mathrm{g}$ were obtained when the ratio of carbon in the material was 0.6 to 0.8 . Characterization of the material after the loading of the $\mathrm{N}-\mathrm{S}-\mathrm{TiO}_{2}$ photocatalyst showed that the surface of the nanocatalyst exhibited a highly ordered lamellar structure. In the future, more research on the catalytic performance of the new material should be conducted.

\section{Acknowledgement}

This work was supported by the National Natural Science Foundation of China (Grant Nos. U1704123 and U1704126) and the Henan Science and Technology Project (172102210224).

\section{References}

1. M.C. Liu, L.B.Kong, P. Zhang, Y.C. Luo, L. Kang, Electrochim Acta, 60 (2012)

2. U. Thubsuang1, S. Laebang, N. Manmuanpom, S. Wongkasemjit, T. Chaisuwan, J mater Sci,52(2017)

3. K.S. Yang, B.H. Kim, Electrochim Acta 186 (2015)

4. D. P. Vargas , L. Giraldo, A. Erto, J. C. MorenoPiraja'n, J Therm Anal Calorim, 114(2013)

5. W. Libbrecht, A. Verberckmoes, J. W. Thybaut, P. V. D. Voort, J. D. Clercq, Carbon, 116 (2017)

6. Y.R. Liu, J Porous Mater, 21 (2014)

7. M.R.M. Jasni, M. Deraman, M. Suleman, Z. Zainuddin, M.A.R. Othman, C.H.Chia, M.A. Hashim, Ionics, 24 (2018)

8. Q.H. Bai, Q.C Xiong, C. Li, Y.H. Shen, H. Uyama, Cellulose, 24 (2017)

9. L. Shoko, J.P. Beukes, C.A. Strydom, B. Larsen, L. Lindstad, Int J Miner Process, 144(2015)

10. HK Park, BS Kong, ES Oh, Electrochem Commun, 13(2011)

11. G.Z. Zhu, X.L. Deng, M. Hou, K. Sun, Y.P. Zhang, P. Li, F.M.Liang, Fuel Process technol, 144(2016)

12. J.K. Song, X.J. Wang, Y.J. Bu, X. Wang, J. Zhang, J.Y. Huang, R.R. Ma, J.F. Zhao, Appl Surf Sci, 391 (2017)

13. Y. Guo, R.X. Wang, P.F. Wang, Y. Li, C. Wang, Chemosphere, 179 (2017)

14. J. Zhang, X. Wang, X. Wang, J. Song, J. Huang, B. Louangsouphom, J. Zhao, RSC Adv, 5 (2015)

15. Y. Li, Y. Huang, Z. Zhang, D. Duan, 272 X. Hao, S. Liu, Chem Eng J, 283(2016)

16. Li, H. Xi, S. Zhu, Z. Wen, R. Wang, Micropor Mesopor Mat, 96 (2016)

17. H. Zhang, L. Chen, L. Li, Y. Yang, X. Liu, J Porous Mater, 24 (2017)

18. L.Z. Zhai, G. Li, Adv Mater Res, 864-867(2014)

19. D.W. Li, J.J. Zhou, Z. B. Zhang, Y.Y.Tian, Y.Y.Qiao, J.H. Li, L. Wen, L. wei , Mater Let, 190 (2017)

20. J.L. Sotelo, G. Ovejero, A. Rodríguez, S. Álvarez, J. García, Water Air SoilPoll. 224(2013)

21. J.K. Song, X.J. Wang, Y.J. Bu, J. Zhang, X. Wang, J.Y. Huang, J. Chen, J.F. Zhao, Environ Sci Pollut Res, 23 (2016)

22. L. Liu, Int.J Electrochem Sci, 12(2017)

23. D.W. Li, J.J. Zhou, Z.B. Zhang, Y.Y. Tian, Y.Y. Qiao, J.H. Li, L. Wen, L. Wei, Mater. Lett., 190 (2017)

24. L. Liu, L. Yang, P. Wang, C.Y. Wang, J. Cheng, G. Zhang, J.J. Gu, F.F. Cao, Int. J. Electrochem. Sci, 12 (2017) 